\title{
4-Dimensional MRI and Attenuation Map Generation in PET/MRI with 4-Dimensional PET-Derived Deformation Matrices: Study of Feasibility for Lung Cancer Applications
}

\author{
Hadi Fayad ${ }^{1}$, Holger Schmidt ${ }^{2}$, Thomas Küstner ${ }^{2,3}$, and Dimitris Visvikis ${ }^{1}$

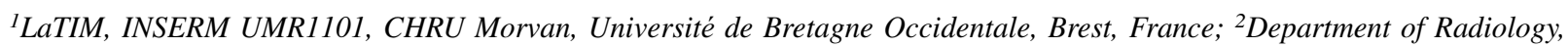 \\ University of Tübingen, Tübingen, Germany; and ${ }^{3}$ University of Stuttgart, Stuttgart, Germany
}

Respiratory motion may reduce accuracy in the fusion of functional and anatomic images from combined PET/MRI systems. Methodologies for the correction of respiratory motion in PET acquisitions with such systems are mostly based on the use of respirationsynchronized MRI acquisitions to derive motion fields. Existing approaches based on tagging acquisitions may introduce artifacts in MR images, whereas motion model approaches require the acquisition of training datasets. The objective of this work was to investigate the possibility of generating 4-dimensional (4D) MR images and associated attenuation maps (AMs) from the combination of a single static MR image and motion fields obtained from simultaneously acquired 4D non-attenuation-corrected (NAC) PET images. Methods: Four-dimensional PET/MRI datasets were acquired for 11 patients on a simultaneous PET/MRI system. The 4D PET datasets were retrospectively binned into 4 motion amplitude frames corresponding to the simultaneously acquired T1-weighted 4D MR images. A T1-weighted 3-dimensional MRI sequence with Dixon-based fat and water separation was also acquired at the end of expiration for PET attenuation correction purposes. All reconstructed 4D NAC PET images were then elastically registered to the single end-ofexpiration NAC PET image. The derived motion fields were subsequently applied to the end-of-expiration frame of the acquired 4D MRI volume and the AM derived from the Dixon MR image to generate respirationsynchronized MR images and corresponding AMs. Results: The accuracy of the proposed method was assessed by comparing the generated and acquired images according to metrics such as overall correlation coefficients and differences in distances of anatomic landmarks on the generated and acquired MRI datasets. High correlation coefficients (mean \pm SD: $0.93 \pm 0.03$ ) and small differences $(2.69 \pm 0.5 \mathrm{~mm})$ were obtained. Moreover, small tissue classification differences $(2.23 \% \pm$ $0.68 \%$ ) between generated and 4D MRI-extracted AMs were observed. Conclusion: Our results confirm the feasibility of using 4D NAC PET images for accurate PET attenuation correction and respiratory motion correction in PET/MRI, without the need for patient-specific 4D MRI acquisitions.

Key Words: 4D PET/MRI; respiratory motion correction; attenuation correction; image generation

J Nucl Med 2017; 58:833-839

DOI: 10.2967/jnumed.116.178947

Received May 31, 2016; revision accepted Sep. 28, 2016.

For correspondence or reprints contact: Hadi Fayad, LaTIM, INSERM

UMR1101, CHRU Morvan, 2 Avenue Foch, 29609 Brest Cedex, France.

E-mail: fayad@univ-brest.fr

Published online Oct. 13, 2016.

COPYRIGHT (C 2017 by the Society of Nuclear Medicine and Molecular Imaging.
$\mathbf{P}$ ET/MRI is a new imaging technique allowing for the simultaneous acquisition of PET and MR images (1). Several challenges associated with the use of MRI for the correction of different effects that reduce overall PET image qualitative and quantitative accuracy, such as scatter, attenuation, and partial-volume effects, were recently identified (2). Moreover, the fusion accuracy of functional and anatomic information may be compromised by patient respiratory motion (3). Developing and combining correction approaches are challenging. The objective of this work was to address such issues with a combination of attenuation correction (AC) and respiratory motion correction.

Current methods for $\mathrm{AC}$ in the thoracic region include the use of a 2-point Dixon acquisition $(4,5)$ that can be exploited to classify tissues into 2-4 different classes (air, fat, soft tissue, and lung) and assign for each class a dedicated attenuation factor. However, this method omits the attenuation caused by bone structures. Other segmentation approaches, such as the use of ultrashort-echo-time MRI sequences $(6,7)$ to account for bone structures by classifying tissues into air, fat, soft tissue, and bone, have been proposed. An alternative methodology consists of atlas-based approaches (8) with a database of registered CT and MRI acquisitions. The challenge with such an approach concerns the ability of the trained model to handle the large variability of pathologic structures compared with the datasets included in the database.

Respiratory motion leads to reductions in both the quantitative accuracy and the qualitative accuracy of PET images. Correction methodologies involve the use of gated PET frames that have a low signal-to-noise ratio because each frame contains only part of the counts available throughout the acquisition of a respirationaveraged PET study (9). More advanced correction approaches involve either correcting for the motion in the image space $(10,11)$ or incorporating motion parameters into the iterative reconstruction process to produce a single motion-compensated PET image $(11,12)$.

Motion parameters may be extracted from 4-dimensional (4D) MR images - which, in principle, can correspond perfectly to PET frames in simultaneous PET/MRI (13) - through the use of temporal information during the MRI acquisition and PET list-mode datasets containing temporal information for each acquired coincidence. However, this theoretic coregistration of PET and MRI data will, in reality, be compromised by imperfect shimming or differences in the durations of the PET and MRI acquisition protocols. In addition, the acquisition of 4D MRI datasets may not always be compatible with clinical PET/MRI practice. On the other hand, the use of 4D pulse sequences and parallel 
reconstruction algorithms is associated with a compromise between image quality and overall acquisition times. This compromise, which is also true for 3-dimensional (3D) MRI acquisitions, becomes more complicated for 4D MRI because of the longer acquisition times involved.

Previously proposed solutions include the use of tagged MRI acquisitions (14) - which may, however, introduce artifacts that reduce the diagnostic quality of acquired MR images. An alternative approach consists of modeling respiratory motion by creating patient-specific (15-17) or generic (18) motion models. These models estimate internal respiratory motion from dynamic 3D MR images and relate this internal motion to surrogate measures extracted with data-driven techniques based on 2-dimensional (2D) or 3D MR image navigators. A disadvantage of these models is the necessary large training datasets.

The main objective of this work was to generate 4D MR images and associated 4D AMs for PET motion correction from the combination of a single static MR image and deformation matrices obtained from simultaneously acquired 4D non-attenuation-corrected (NAC) PET images. A similar concept was previously proposed for 4D PET/CT imaging (18). The aim was to replace lengthy 4D MRI acquisitions (13-17) with a single static MRI acquisition and facilitate accurate 4D MRI-based AC for the simultaneously acquired 4D PET datasets by generating MR image series that mimicked 4D MRI acquisitions. The availability of $4 \mathrm{D}$ attenuation-corrected PET respiratory gated images in which all of the statistics acquired throughout the respiratory cycle are used in each respiratory frame is essential for certain applications, such as the use of PET/MRI in radiotherapy, in which the entire extent of tumor motion is necessary for the implementation of a 4D treatment protocol. The proposed approach was tested with 11 patients who underwent 4D PET/MRI acquisitions on a Siemens Biograph mMR PET/MRI system.

\section{MATERIALS AND METHODS}

\section{General Method Description}

A prerequisite of the proposed approach is the availability of a dynamic respiration-synchronized PET image series and a unique endof-expiration 3D MRI volume. The aim of the proposed method was to generate MR images and their corresponding AMs, associated with each PET respiration-synchronized frame, through several steps (Fig. 1): respiratory gated NAC PET frames were reconstructed, and the one

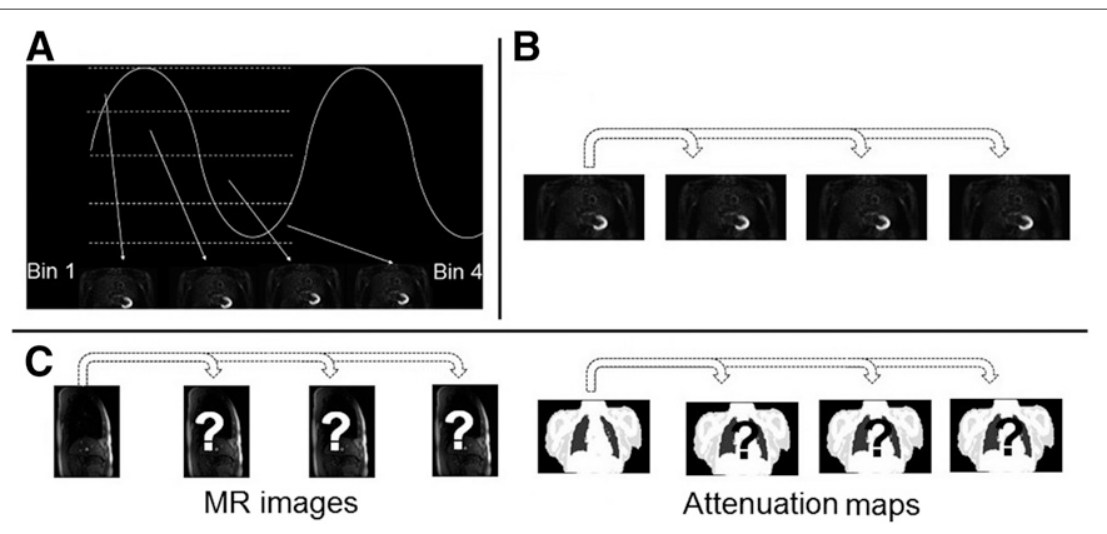

FIGURE 1. Work flow of proposed method. (A) 4D NAC PET reconstruction and gating into 4 bins. (B) Registration of NAC PET bins to reference end-of-expiration bin. (C) Application of obtained motion fields to end-of-expiration 3D MRI volume and corresponding AMs. corresponding to the end-of-expiration state was used as a reference PET frame. The reference PET frame was registered to all NAC PET frames with a nonrigid registration algorithm to derive associated deformation matrices. These matrices were subsequently applied to either the acquired end-of-expiration MRI volume to generate the corresponding respiration-synchronized MRI frames or to the AM extracted from the end-of-expiration 3D Dixon MRI volume to generate $4 \mathrm{D}$ respiration-synchronized AMs.

\section{Patient Datasets}

Eleven consecutively enrolled patients with thoracic or upperabdomen lesions (bronchial, sigma, and nasopharyngeal carcinomas) participated in the study (age: range, 32-70 y; mean $\pm \mathrm{SD}, 58 \pm$ $11 \mathrm{y})$. Table 1 shows an overview of patient demographics, including corresponding lesion characteristics. Patient datasets were acquired on a whole-body PET/MRI system (Siemens Biograph mMR), which combines a PET system with transaxial and axial fields of view of 59.4 and $25.8 \mathrm{~cm}$, respectively, and a 3-T MRI system. Phased-array body coils optimized for, minimally, $511-\mathrm{keV}$ photon attenuation were used for signal detection. A T1-weighted spoiled-gradient-echo sequence with Dixon-based fat and water separation was acquired at breath-hold (echo times, 1.23 and $2.46 \mathrm{~ms}$; repetition time, $3.6 \mathrm{~ms}$; flip angle, $10^{\circ}$; resolution, $2.6 \times 2.6 \times 2.6 \mathrm{~mm}$; bandwidth, $965 \mathrm{~Hz} /$ pixel; parallel imaging acceleration factor, 2; slices per slab, 128; acquisition time, $19 \mathrm{~s}$; end-of-expiration position), and tissues were classified into air, lung, soft tissue, and adipose tissue to obtain an AM for the entire PET field of view $(5,19)$. PET data from 1 bed position (covering the thorax and upper abdomen) were subsequently recorded in the list mode for 5 min under free-breathing conditions. PET/MRI scanning was started without repeated radiotracer injection after routine PET/CT (124 \pm 11 min after injection of $336-371 \mathrm{MBq}$ of $\left.{ }^{18} \mathrm{~F}-\mathrm{FDG}\right)$.

The 1-dimensional respiratory signal used for PET data binning was extracted from 2D MR image navigators at the diaphragm position (13). Finally, during the first $3 \mathrm{~min}$ of the PET scan, multiple sagittal 2D MRI slices covering the PET field of view were acquired to obtain a 4D MRI series for validation purposes (echo time, $1.8 \mathrm{~ms}$; repetition time, $3.7 \mathrm{~ms}$; flip angle, $15^{\circ}$; resolution, $2 \times 2 \times 10 \mathrm{~mm}$; bandwidth, $670 \mathrm{~Hz} /$ pixel; slices per slab, 36; acquisition time per image slice, $0.4 \mathrm{~s}$ ) (13). To reconstruct the 4D MRI series and to cover 1 respiratory cycle, we acquired 12 frames of each slice sequentially before moving to the next slice. Thirty-six slice positions in the leftto-right direction, covering the patient's thorax and upper abdomen, were acquired, resulting in approximately $10-\mathrm{mm}$ resolution in this direction. This low resolution was acceptable considering the lowamplitude respiratory motion generally in this direction (12). The amplitude of the diaphragm position extracted from the MR image navigators was used to define 4 respiratory gates by dividing the range between the 0.05 and 0.95 quantiles into 4 equally sized intervals (to reduce the influence of outliers). The mean temporal respiratory position within a respective gate interval was subsequently determined. Thus, for each slice and each gated list-mode frame, the MR image closest to the mean temporal respiratory position of the frame was inserted into the corresponding 3D MRI volume, and the 11 remaining 2D MR images were discarded for the specific temporal frame (12).

All patients provided written informed consent for participation in the 4D PET/MRI study, which was approved by the local institutional review board. 
TABLE 1

Overview of Patient Demographics, Including Lesion and Associated Motion Characteristics

\begin{tabular}{|c|c|c|c|c|c|c|}
\hline Patient & Age $(y)$ & Sex & Type of malignancy & Lesion location & Volume $\left(\mathrm{cm}^{3}\right)^{\star}$ & Lesion displacement $(\mathrm{mm})^{\star}$ \\
\hline 1 & 67 & M & Bronchial carcinoma & Lymph node, hilar & 0.44 & 5.1 \\
\hline 2 & 61 & M & Gastrointestinal stromal tumor & Stomach & 1.80 & 7.4 \\
\hline 3 & 70 & M & Bronchial carcinoma & Lung, lower lobe & 0.97 & 16.2 \\
\hline 4 & 56 & M & Nasopharyngeal carcinoma & Spleen & 1.16 & 9.3 \\
\hline 5 & 32 & $\mathrm{~F}$ & Sigma carcinoma & Spleen & 1.65 & 13.7 \\
\hline 6 & 69 & M & Bronchial carcinoma & Lung, lower lobe & 0.34 & 13.5 \\
\hline 7 & 62 & $\mathrm{~F}$ & Laryngeal carcinoma & Lung, middle lobe & 0.58 & 5.6 \\
\hline 8 & 60 & $\mathrm{~F}$ & Bronchial carcinoma & Lung, lower lobe & 0.31 & 7.4 \\
\hline 9 & 49 & M & Esophageal carcinoma & Middle thoracic esophagus & 1.27 & 7.8 \\
\hline 10 & 54 & M & Bronchial carcinoma & Lung, lower lobe & 0.53 & 10.4 \\
\hline 11 & 52 & $\mathrm{~F}$ & Sigma carcinoma & Lung, lower lobe & 0.66 & 11 \\
\hline
\end{tabular}

*Determined by experienced radiologist using manual selection on 4D MR images.

\section{Dataset Preparation}

For every patient, the acquired 4D PET datasets were retrospectively binned into 4 motion amplitude frames corresponding to the simultaneously acquired 4D MR images and reconstructed with a 3D ordered-subsets expectation maximization algorithm $(20,21)$ with 3 iterations, 21 subsets, a 5-mm gaussian filter, and a voxel size of $1.78 \times 1.78 \times 2 \mathrm{~mm}$ but without AC. The choice of 4 frames was based on previous work in which it was shown that for a 5-min PET acquisition with the mMR system, 4 amplitude-binned gates resulted in better lesion contrast, signal-to-noise ratio, and full width at half maximum results than 6 or 8 gates (13).

\section{Image Generation}

All reconstructed 4D NAC PET images were registered to the single end-of-expiration NAC PET image corresponding to the end-ofexpiration frame of the acquired 4D MRI volume with a B-splinebased deformable registration algorithm (22), previously validated for use with PET (12). The influence of the PET image statistics on the registration results was evaluated as previously described and shown to be negligible (23).

The derived motion fields were subsequently applied to the end-ofexpiration frame of the acquired 4D MRI volume and the corresponding AM to generate respiration-synchronized MR images and corresponding AMs, respectively. For the acquisition of a single MR image for each patient, the synchronized respiratory signal can be used to determine the corresponding temporal and motion amplitude information. Subsequently, with the PET list-mode data and the temporal/amplitude characteristics of the single acquired MRI frame, a corresponding PET image can be reconstructed (reference PET frame).

For application of the motion fields to the single acquired MRI frame (or the corresponding $\mathrm{AM}$ ), $f(x)$, it is necessary to approximate the B-spline coefficients derived from the nonrigid registration into discrete 3D voxel-based displacement vector images. To avoid such approximations, we instead used a $2 \mathrm{D}$ spline interpolation to represent the MRI frame, $f(x)$, as:

$$
f(x)=\sum_{i \in Z^{2}} b_{i} \beta^{r}(x-i),
$$

where $\beta^{r}(x)$ is a tensor product of centered B-splines of degree $r$ and coefficient $b_{i}$ is obtained from voxel values $f(i)$ through filtering in the rectangular domain $Z^{2}$. The set of $2 \mathrm{D}$ spline interpolations can represent a 3D object corresponding to an MRI frame. We chose 2D splines because they are the smoothest of all possible interpolating curves

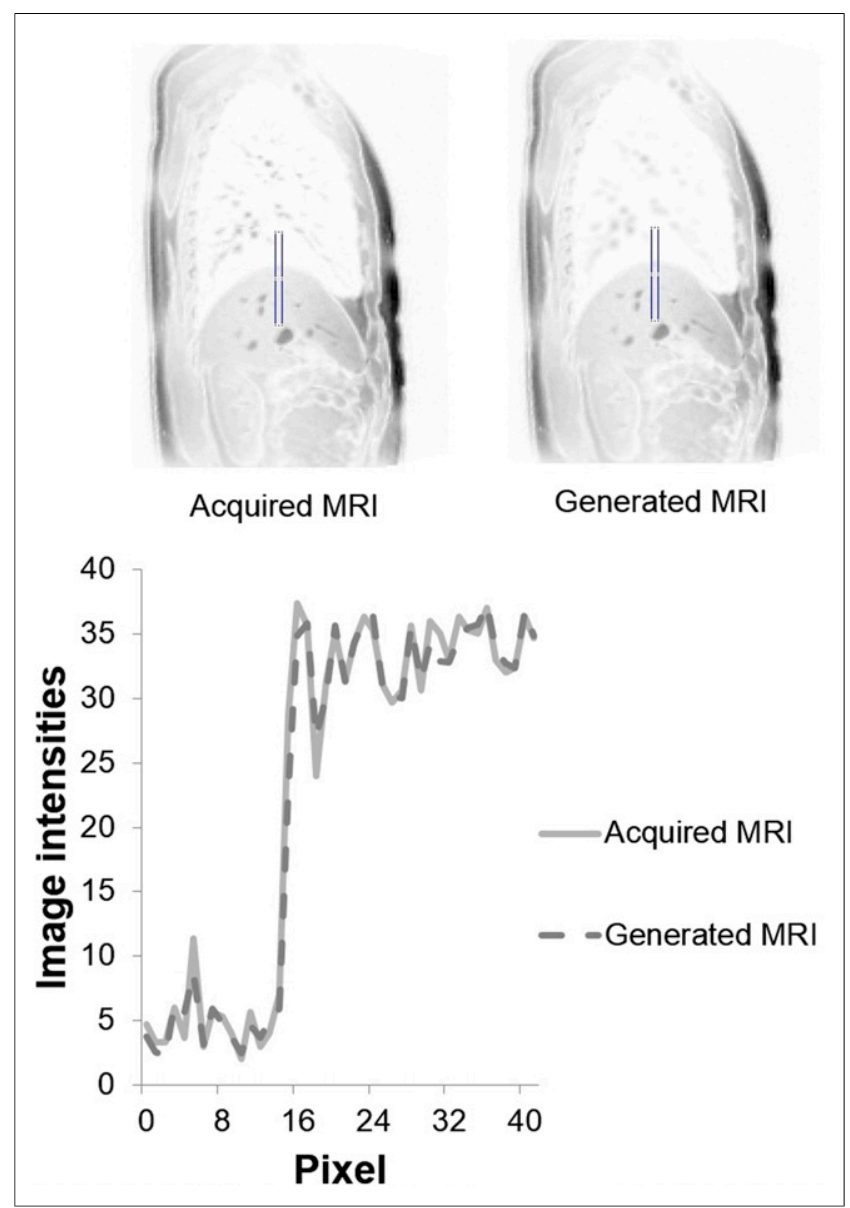

FIGURE 2. Profile drawn across diaphragm on both acquired and generated MR images (sagittal view). 


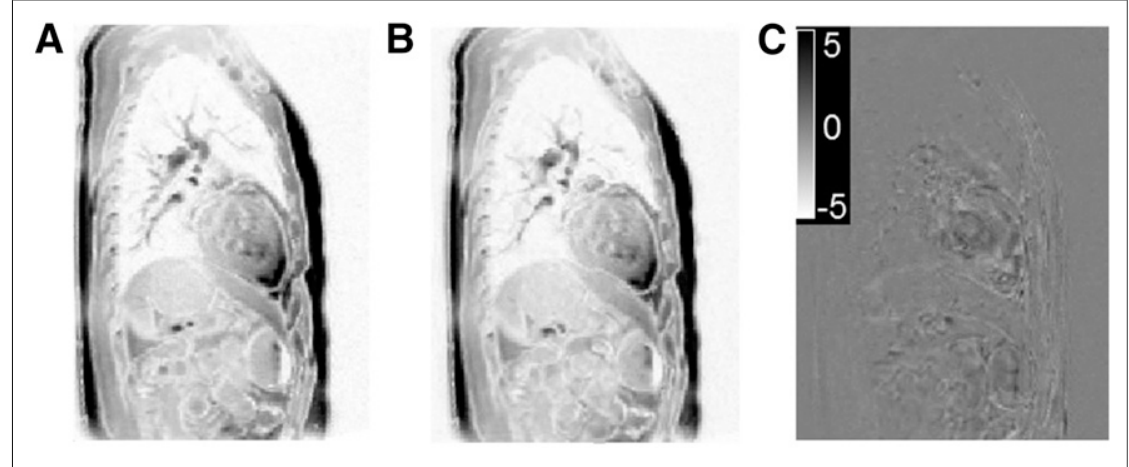

FIGURE 3. (A) Sagittal view of slice of acquired MR image. (B) Sagittal view of same slice of corresponding generated MR image. (C) Difference between 2 images.

(such as polynomials) in the sense that they minimize the integral of the square of the second derivative and their superiority over other alternatives was previously proved (12). Finally, the warped sequence, $f\left(U_{r-s}\right)$ (acquired 4D MRI or AM sequence), was generated by applying the deformation matrices, $U_{r-s}$ (where $r$ and $s$ correspond to the reference frame and one of the respiration-synchronized NAC PET frames, respectively), to $f(x)$.

\section{Image Analysis and Validation}

The accuracy of the proposed method was assessed by comparing the generated 4D MR images with the originally acquired 4D MR images. Regarding the AMs, we compared those generated with the proposed method and the corresponding ones extracted from the 4D MRI series by using the 3D Dixon MRI sequence corresponding to the end-of-expiration PET frame as a reference.

Local image analysis was performed with profiles placed across moving parts of the patient's anatomy, such as the diaphragm. A qualitative global comparison was performed with difference images. For global quantitative estimation, we computed a correlation coefficient, $\rho$, which measured a linear affine relationship between the intensities of the 2 images being compared $(A$ and $B)$ :

$$
\rho_{(A, B)}=\sum_{a} \sum_{b} \frac{\left(I_{a}-M_{A}\right)\left(I_{b}-M_{B}\right)}{\sqrt{\sigma_{A}} \sqrt{\sigma_{B}}},
$$

Eq. 2

where $I_{a}$ and $I_{b}$ are the intensities of voxels $a$ of image A and voxels $b$ of image $\mathrm{B}$, respectively $(0<a<$ number of voxels of image A; $0<$ $b<$ number of voxels of image B), and $M$ and $\sigma$ are the mean and SD of the image intensities. The closer $\rho$ is to 1 , the stronger the positive correlation.

Finally, a validation based on landmark identification was performed (24). Two radiologists with more than $15 \mathrm{y}$ of experience in MRI selected the same easily identifiable anatomic landmarks on both the acquired 4D MR images (ground truth) and the generated 4D MRI volumes. The Euclidian distance (ED) between points in the reference volume and the warped MRI volumes was subsequently calculated:

$$
\mathrm{ED}=\frac{1}{N}\left(\left\|q_{k}-r_{k}\right\|^{2}\right)
$$

where $q_{k}$ and $r_{k}$ are the coordinates of the $k^{\text {th }}$ landmark in the acquired and generated MR images, respectively. ED is expressed in millimeters, and $N$ is the total number of anatomic landmarks considered. Thirteen landmarks covering different thoracic areas were used to investigate regions of interest characterized by variable magnitudes of motion due to respiration (25-28). These included the right and left apices; aortic cross; sternum; carina; left and right nipples; high generated 4D MRI series.

positions of the left diaphragm and right diaphragm; and high, low, left, and right boundaries of the tumor (29).

In addition, a region-of-interest analysis in the lungs was used to compare the different attenuation-corrected PET images obtained with the original and warped 4D MRI series and their corresponding AMs.

\section{RESULTS}

Figure 2 shows examples of an acquired MR image (corresponding to the ground truth) and a generated MR image. Profiles along the diaphragm (illustrated at the bottom of Fig. 2 for one patient) indicated a good match between the acquired and the

Figure 3 shows the differences between the acquired and the generated MR images; the differences were small $(-5$ to +5 , representing differences of $<2 \%$ given that the images were normalized between 0 and 255). For the 11 patients, high correlation coefficients (overall mean $\pm \mathrm{SD}, 0.93 \pm 0.03$ ) between both series were measured, as shown in Table 2.

Figure 4A shows the mean ED results and the corresponding SDs for the acquired and generated MR images (for all respiratory phases in each patient). The mean ED was $2.69 \mathrm{~mm}$ (SD, $0.5 \mathrm{~mm}$ ), and the maximum ED was $3.38 \mathrm{~mm}$ (Fig. 4B, box-and-whisker plot). Moreover, the repeatability results for identifying the internal anatomic landmarks revealed a mean intraoperator and interoperator variability of less than $1.5 \mathrm{~mm}$, which demonstrated good intraoperator agreement in the identification of landmarks.

Finally, the acquired MRI series were used to create 4D respiration-synchronized AMs with a 2-point Dixon approach. At each step, we compared AMs generated with our method and those extracted from the 4D MRI series. Figure 5 shows axial, coronal, and sagittal views of an AM generated with our method

\section{TABLE 2}

Coefficients of Correlation Between Acquired and Generated MR Images for 11 Patients

\begin{tabular}{lc}
\hline Patient & $\begin{array}{c}\text { Correlation coefficient } \\
(\text { mean } \pm \text { SD) }\end{array}$ \\
\hline 1 & $0.954 \pm 0.013$ \\
2 & $0.963 \pm 0.021$ \\
3 & $0.927 \pm 0.037$ \\
4 & $0.892 \pm 0.035$ \\
5 & $0.915 \pm 0.026$ \\
6 & $0.981 \pm 0.007$ \\
7 & $0.921 \pm 0.019$ \\
\hline 9 & $0.892 \pm 0.034$ \\
\hline 10 & $0.924 \pm 0.029$ \\
11 & $0.912 \pm 0.027$ \\
\hline
\end{tabular}

*Mean \pm SD corresponds to 3 predicted volumes for each patient. 


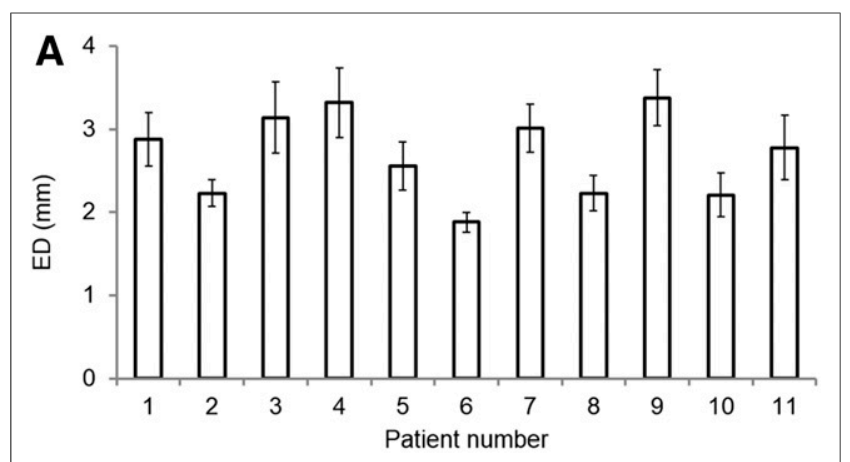

B

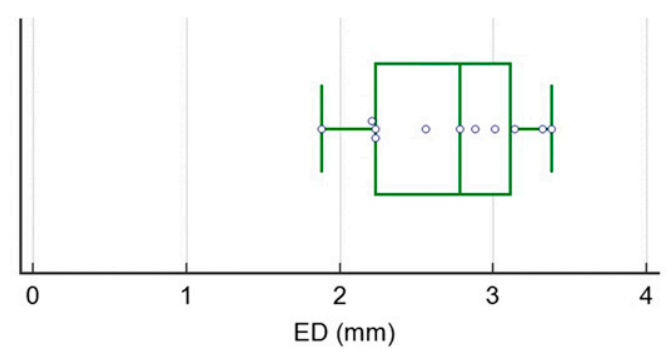

FIGURE 4. (A) Comparison of ED (in $\mathrm{mm}$ ) and corresponding SD for all anatomic landmarks considered in all 11 patient datasets. (B) Box-andwhisker plot for ED (in $\mathrm{mm}$ ) in all 11 patient datasets.

(Fig. 5A), the corresponding 4D MRI-generated AM (Fig. 5B), and the difference between the images (Fig. 5C) - in which black (intensity, 0) and white (intensity, 1) voxels corresponded to correct and incorrect classifications, respectively. There was a high correlation between maps generated with our method and those extracted from the 4D MRI series (Table 3); the mean correlation coefficient for all 11 patients was 0.93 (SD, 0.03). Figure 6A shows the percentages of the voxels that were misclassified, with a mean error of 2.23 (Fig. 6B, box-and-whisker plot).

The region-of-interest analysis of the attenuation-corrected PET images with the original and warped MRI-generated AMs for all patients revealed a mean difference of $2.65 \%$, with corresponding intensities (radioactivity concentrations) of $18.22 \pm 3.16$ and $17.39 \pm 3.85 \mathrm{MBq} / \mathrm{mL}$ for the original and warped MRI-generated AMs, respectively.

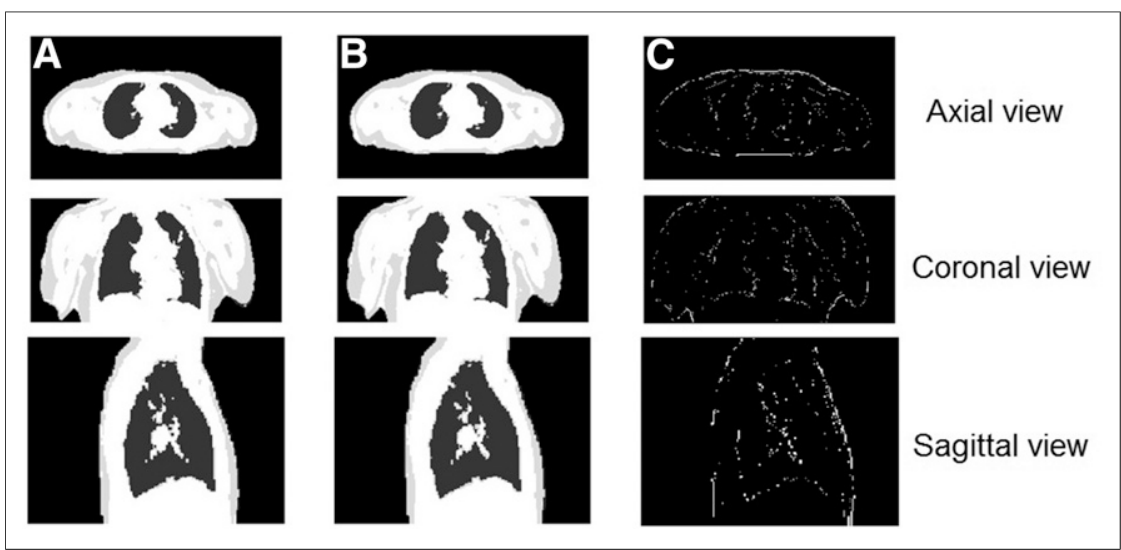

FIGURE 5. (A) Axial, coronal, and sagittal views of AM generated with our method based on 4tissue-class attenuation model. (B) Corresponding 4D MRI-generated AM based on 4-tissueclass attenuation model. (C) Difference between 2 AMs.

\section{DISCUSSION}

The goal of the present study was to investigate the feasibility of producing a 4D MRI series from corresponding 4D NAC PET images and a single MRI acquisition, thus eliminating the need for 4D MRI acquisitions. Eleven patient datasets were included in the validation. The generated and acquired MRI series were highly correlated. The results demonstrated the ability of the proposed method to derive 4D MRI frames from a 4D NAC PET image registration and a reference MRI frame. In addition, the generated MRI frames could create 4D AMs to improve MRI-based AC in 4D PET imaging, in terms of correspondence between the 2 datasets (same motion characteristics). For all 11 patients, the generated and acquired AMs were well correlated, as were the corresponding attenuation-corrected PET images obtained with these AMs. Although this methodology was previously evaluated for PET/CT imaging (23), the present study demonstrated that it is also applicable for PET/MRI, reducing the need for 4D MRI acquisitions for 4D PET AC.

In addition, within the context of PET/MRI respiratory motion correction, this approach may resolve issues associated with the acquisition of motion-monitoring specific MRI sequences (16), reducing the overall time available for clinically relevant MRI data. The respiratory motion correction can be based on the use of the deformation fields applied in the present work for 4D MRI and AM generation, either in the image space (10) or within the iterative reconstruction process, to produce a single motion-compensated PET image (12). Therefore, this approach allows parallel attenuation and respiratory motion correction and may be comparable to alternative methodologies based on patient-specific (15-17) or generic (18) motion models relating internal respiratory motion from dynamic 3D MR images to surrogate measures extracted with datadriven techniques based on 2D or 3D MR image navigators.

A possible limitation of the present study is that a unique level of PET image statistical quality was considered (5-min acquisition per bed position). However, in a previous study concerning PET/ CT imaging (23), different levels of PET image statistical quality were considered, and the results demonstrated that clinically relevant PET acquisition times ( 3 min per bed position) were sufficient for the implementation of such an approach. Another limitation of the present study is that the use of a 2-point Dixon approach for AC omits the attenuation caused by bone structures. For resolution of this issue, different approaches-such as the use of ultrashort-echo-time MRI sequences (30) and combining ultrashort-echo-time sampling for bone detection and gradient echoes for Dixonbased water and fat separation in a radial 3D acquisition-were recently proposed. However, given that the proposed approach can generate 4D MR images and corresponding AMs, it can be easily extended to take into account bones when ultrashort-echo-time or alternative MRI sequences are used for PET AC purposes.

Finally, a potential improvement of the proposed methodology would be the use of time-of-flight PET acquisitions, which might help improve the quality of NAC PET images and therefore improve the accuracy and robustness of corresponding deformation matrices. 
TABLE 3

Coefficients of Correlation Between AMs Generated with Proposed Method and Those Extracted from 4D MRI Series for 11 Patients

\begin{tabular}{lc}
\hline Patient & $\begin{array}{c}\text { Correlation coefficient } \\
(\text { mean } \pm \text { SD) }\end{array}$ \\
\hline 1 & $0.982 \pm 0.007$ \\
\hline 2 & $0.978 \pm 0.011$ \\
3 & $0.955 \pm 0.016$ \\
\hline 4 & $0.876 \pm 0.042$ \\
5 & $0.909 \pm 0.033$ \\
6 & $0.973 \pm 0.012$ \\
\hline 7 & $0.928 \pm 0.023$ \\
\hline 9 & $0.937 \pm 0.031$ \\
10 & $0.887 \pm 0.04$ \\
11 & $0.891 \pm 0.025$ \\
\hline
\end{tabular}

*Mean \pm SD corresponds to 3 predicted volumes for each patient.

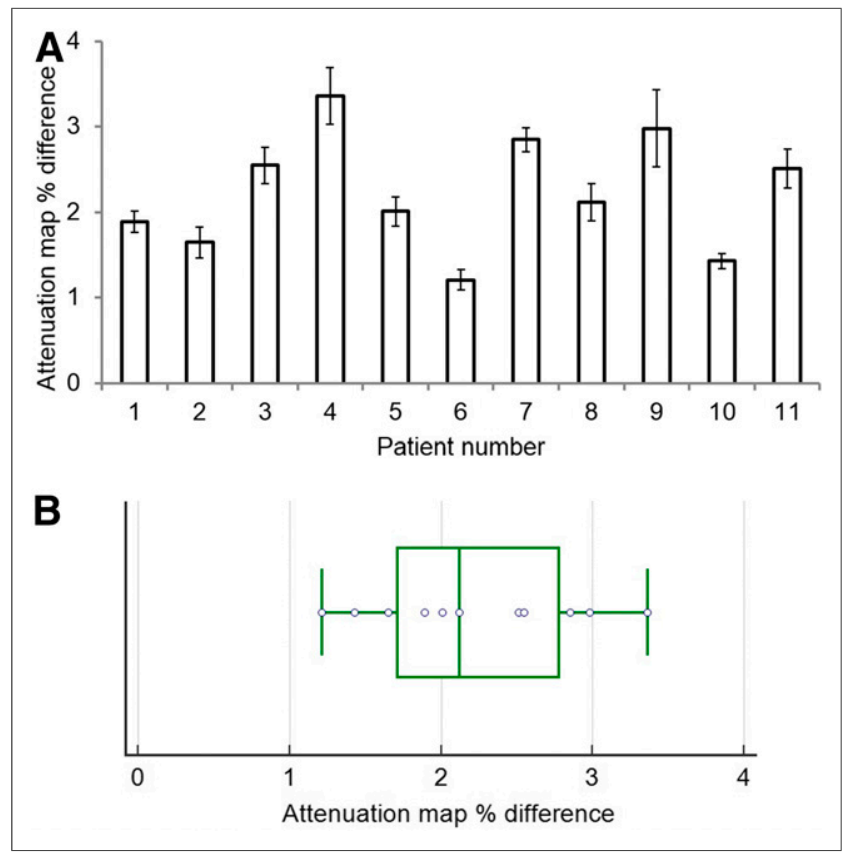

FIGURE 6. (A) AM percentage difference and corresponding SD in all 11 patient datasets. (B) Box-and-whisker plot for AM percentage difference in all 11 patient datasets.

\section{CONCLUSION}

The present study demonstrated the feasibility of using 4D NAC PET images to derive matching 4D MRI series and corresponding AMs from a single-respiratory-state MR image. The efficiency was assessed with 11 clinical patient datasets. The results demonstrated a high correlation between generated and acquired MRI series and corresponding AMs, thus eliminating the need for long 4D MRI acquisitions. Future work will consist of exploring the proposed approach in conjunction with respiratory motion models to reduce the potential limitation of monitoring noncyclic motion with only the 4 respiratory gates used in the present study.

\section{DISCLOSURE}

No potential conflict of interest relevant to this article was reported.

\section{REFERENCES}

1. Delso G, Fürst S, Jakoby B, et al. Performance measurements of the Siemens mMR integrated whole-body PET/MR scanner. J Nucl Med. 2011;52:19141922.

2. Bailey DL, Antoch G, Bartenstein P, et al. Combined PET/MR: the real work has just started-summary report of the Third International Workshop on PET/MR Imaging; February 17-21, 2014, Tübingen, Germany. Mol Imaging Biol. 2015;17:297-312.

3. Brendle CB, Schmidt H, Fleischer S, Braeuning UH, Pfannenberg CA, Schwenzer NF. Simultaneously acquired MR/PET images compared with sequential MR/PET and PET/CT: alignment quality. Radiology. 2013;268: 190-199.

4. Dixon WT. Simple proton spectroscopic imaging. Radiology. 1984;153: 189-194.

5. Martinez-Möller A, Souvatzoglou M, Delso G, et al. Tissue classification as a potential approach for attenuation correction in whole-body PET/MRI: evaluation with PET/CT data. J Nucl Med. 2009;50:520-526.

6. Catana C, van der Kouwe A, Benner T, et al. Toward implementing an MRIbased PET attenuation-correction method for neurologic studies on the MR-PET brain prototype. J Nucl Med. 2010;51:1431-1438.

7. Keereman V, Fierens Y, Broux T, Deene YD, Lonneux M, Vandenberghe S. MRI-based attenuation correction for PET/MRI using ultrashort echo time sequences. J Nucl Med. 2010;51:812-818.

8. Hofmann M, Steinke F, Scheel V, et al. MRI-based attenuation correction for PET/MRI: a novel approach combining pattern recognition and atlas registration. J Nucl Med. 2008;49:1875-1883.

9. Bruyant P, Turzo A, Bizais Y, Cheze Le Rest C, Visvikis D. A comparison of three respiratory gating methods in PET imaging for oncology [abstract]. $\mathrm{J} \mathrm{Nucl}$ Med. 2006;47:183Pa.

10. Dawood M, Lang N, Jiang X, Schafers KP. Lung motion correction on respiratory gated 3-D PET/CT images. IEEE Trans Med Imaging. 2006;25: 476-485.

11. Fayad H, Schmidt H, Würslin C, Visvikis D. Reconstruction incorporated respiratory motion correction in clinical simultaneous PET/MR imaging for oncology applications. J Nucl Med. 2015;56:884-889.

12. Lamare F, Ledesma Carbayo MJ, Cresson T, et al. List-mode-based reconstruction for respiratory motion correction in PET using non-rigid body transformations. Phys Med Biol. 2007;52:5187-5204.

13. Würslin C, Schmidt H, Martirosian $\mathrm{P}$, et al. Respiratory motion correction in oncologic PET using T1-weighted MR imaging on a simultaneous whole-body PET/MR system. J Nucl Med. 2013;54:464-471.

14. Guérin B, Cho S, Chun SY, et al. Nonrigid PET motion compensation in the lower abdomen using simultaneous tagged-MRI and PET imaging. Med Phys. 2011;38:3025-3038.

15. Fayad H, Odille F, Schmidt H, et al. The use of a generalized reconstruction by inversion of coupled systems (GRICS) approach for generic respiratory motion correction in PET/MR imaging. Phys Med Biol. 2015;60: 2529-2546.

16. King AP, Buerger C, Tsoumpas C, Marsden PK, Schaeffter T. Thoracic respiratory motion estimation from MRI using a statistical model and a 2-D image navigator. Med Image Anal. 2012;16:252-264.

17. Dikaios N, Izquierdo-Garcia D, Graves MJ, Mani V, Fayad ZA, Fryer TD. MRIbased motion correction of thoracic PET: initial comparison of acquisition protocols and correction strategies suitable for simultaneous PET/MRI systems. Eur Radiol. 2012;22:439-446.

18. Fayad HJ, Buerger C, Tsoumpas C, Cheze-Le-Rest C, Visvikis D. A generic respiratory motion model based on 4D MRI imaging and 2D image navigators. In: 2012 IEEE Nuclear Science Symposium and Medical Imaging Conference (NSS/MIC). New York, NY: Institute of Electrical and Electronics Engineers; 2012:4058-4061. 
19. Bezrukov I, Schmidt H, Gatidis S, et al. Quantitative evaluation of segmentationand atlas-based attenuation correction for PET/MR on pediatric patients. $\mathrm{J} \mathrm{Nucl}$ Med. 2015;56:1067-1074.

20. Hudson HM, Larkin RS. Accelerated image reconstruction using ordered subsets of projection data. IEEE Trans Med Imaging. 1994;13:601-609.

21. Liu X, Comtat C, Michel C, Kinahan P, Defrise M, Townsend D. Comparison of 3-D reconstruction with 3D-OSEM and with FORE+OSEM for PET. IEEE Trans Med Imaging. 2001;20:804-814.

22. Ledesma-Carbayo MJ, Mahía-Casado P, Santos A, Pérez-David E, GarcíaFernández MA, Desco M. Cardiac motion analysis from ultrasound sequences using nonrigid registration: validation against Doppler tissue velocity. Ultrasound Med Biol. 2006;32:483-490.

23. Fayad HJ, Lamare F, Le Rest CC, Bettinardi V, Visvikis D. Generation of 4dimensional CT images based on 4-dimensional PET-derived motion fields. $J$ Nucl Med. 2013;54:631-638.

24. Sarrut D, Boldea V, Miguet S, Ginestet C. Simulation of four-dimensional CT images from deformable registration between inhale and exhale breath-hold CT scans. Med Phys. 2006;33:605-617.
25. Giraud P, Reboul F, Clippe S, et al. Respiration-gated radiotherapy: current techniques and potential benefits [in French]. Cancer Radiother. 2003;7(suppl 1): $15 \mathrm{~s}-25 \mathrm{~s}$.

26. Murphy MJ, Martin D, Whyte R, Hai J, Ozhasoglu C, Le Q-T. The effectiveness of breath-holding to stabilize lung and pancreas tumors during radiosurgery. Int $J$ Radiat Oncol Biol Phys. 2002;53:475-482.

27. Ionascu D, Jiang SB, Nishioka S, Shirato H, Berbeco RI. Internal-external correlation investigations of respiratory induced motion of lung tumors. Med Phys. 2007;34:3893-3903.

28. van Sörnsen de Koste JR, Lagerwaard FJ, Nijssen-Visser MRJ, Graveland WJ, Senan S. Tumor location cannot predict the mobility of lung tumors: a 3D analysis of data generated from multiple CT scans. Int J Radiat Oncol Biol Phys. 2003;56:348-354.

29. Fayad H, Pan T, Clement JF, Visvikis D. Technical note: correlation of respiratory motion between external patient surface and internal anatomical landmarks. Med Phys. 2011;38:3157-3164.

30. Berker Y, Franke J, Salomon A, et al. MRI-based attenuation correction for hybrid PET/MRI systems: a 4-class tissue segmentation technique using a combined ultrashort-echo-time/Dixon MRI sequence. J Nucl Med. 2012;53: 796-804. 